\title{
Adapting ADDIE Model for Human Robot Interaction in Soccer Robotics Domain
}

\author{
Rajesh Elara Mohan, Carlos A. Acosta Calderon, Changjiu Zhou, Tianwu Yang, \\ Liandong Zhang, and Yongming Yang \\ Advanced Robotics and Intelligent Control Centre, \\ School of Electrical and Electronics Engineering, \\ Singapore Polytechnic, 500 Dover Road, Singapore \\ \{ZhouCJ, MohanRajesh\} @ sp.edu.sg \\ www.robo-erectus.org
}

\begin{abstract}
Though human robot interaction has attracted lots of attention in the recent years due to increasing presence of robots in the marketplace, very little work has been done with systematic human robot interaction development using process models. Analysis, Design, Develop, Implement and Evaluate (ADDIE) model is one of the well established systematic process models for developing instructions in instructional design (ID) community. This paper focuses on two issues: 1) guidance in adapting ADDIE model for human robot interaction, and 2) performance improvement in adopting ADDIE model for human robot interaction in soccer robotics domain. Evaluations were performed and adequate results were obtained. ADDIE modelled human robot interaction was used by our Robo-Erectus Junior humanoid robots in the 2 versus 2 humanoid leagues of RoboCup 2007.
\end{abstract}

\section{Introduction}

Growing popularity and increasing viable application domains has contributed to greater presence of robots in the commercial marketplace. Among various robotic platforms, especially humanoid robotic research has seen a rapid growth in the recent years due to the ability of humanoid robots to behave and interact like humans. Humanoid robots might provide day-to-day support in the home and the workplace, doing laundry or dishes, assisting in the care of the elderly, or acting as a caretaker for individuals within a home or institution [1]. Many of these tasks will involve a close interaction between the robot and the people it serves. Researchers have studied the humanoid robot development, including control, emotional expressiveness, humanoid-humanoid collaboration, human robot interaction and perception [2][3][4]. However, there have been only fewer studies with systematic human robot interaction development using process models.

The Analysis, Design, Develop, Implement and Evaluate (ADDIE) model is one of the most widely used systematic process model in instructional design community. ADDIE model provides a generic and systematic framework to the instructional 
design process that can be applied to any learning solutions. Most of the currently used instructional design models are simple variants of the ADDIE model. ADDIE model comprises of five elements namely, analysis, design, develop, implement and evaluate. The five elements are ongoing activities that continue throughout the life of an instructional program. After building an instructional program, the other phases do not end once the instructional program is implemented. The five elements work like a loop. They are continually repeated on a regular basis to see if further improvements can be made [5]. ADDIE model being a mature systematic process model can be modified and applied to the human robot interaction development for performance improvement.

Soccer robotics serves as an excellent platform for evaluating performance of an ADDIE modelled human robot interaction. RoboCup is an international joint research initiative to foster artificial intelligence, robotics, and related research. RoboCup uses soccer game as a domain of research, aiming at innovations to be applied for socially significant problems. The ultimate goal of the RoboCup initiative is to develop a team of fully autonomous humanoid robots that can win against the human world champion team in soccer. Our Robo-Erectus has participated in the humanoid league of RoboCup since 2002, collecting several awards since then. Various technologies are incorporated into a soccer robot including, multi robot cooperation, human robot interaction, sensor fusion, artificial intelligence, real time processing, navigation and localization.

Every league has its own requirements on the robot hardware and software systems. Humanoid 2 versus 2 soccer match is played with two teams of two robots in autonomous mode on a $4.5 \mathrm{~m} \times 3 \mathrm{~m}$ field. Each robot must have a human-like body plan with two legs, two arms, and one head, which are attached to a trunk and fit into a cylinder of diameter $0.55^{*} \mathrm{H}$, where $\mathrm{H}$ is the height of the humanoid robot. During the soccer match, system failures occur due to the hardware and software complexity of the humanoid robot operating in autonomous mode. The corresponding team request for stoppage of game with a timeout period of 120 s to find fault and service their robot and the game starts after the elapse of 120s [6]. Therefore, humanoid 2 versus 2 soccer match requires human robot interaction design to be able to detect the system fault with the robot as early as possible. Experimental results have shown that the interaction design must be able to find the fault with the robot in atleast $60 \mathrm{~s}$ or within half the timeout period so as to enable the user to fix the fault within the timeout period. Delay in finding faults during timeout period might provide an edge for the opponent team as they can start the playing game.

This paper focuses on adapting ADDIE model for human robot interaction development in soccer domain and evaluates the performance of the developed interaction. The ADDIE modelled human robot interaction design of Robo-Erectus Junior was evaluated and the results of the experiments showed that, the interaction design was able to find faults in an average time of $22.91 \mathrm{~s}$ as compared to $57.47 \mathrm{~s}$ with the unmodelled interaction design. Also, the interaction design was able to detect the fault within 60 s in $100 \%$ of the cases. Our Robo-Erectus Junior version of humanoid robot 
was equipped with the evaluated interaction design in RoboCup 2007 humanoid soccer league.

Section 2 discusses ADDIE model and its application in human robot interaction, while section 3 provides a brief introduction of our humanoid platform, Robo-Erectus Junior and its ADDIE modelled human robot interaction. Section 4 summarizes the experiments performed and the results. Finally, section 5 presents the conclusion of the paper.

\section{ADDIE Model}

ADDIE model is considered to be the most universal instructional designer model. It is widely used by instructional designers and training developers. The ADDIE model encompasses five elements of the instructional design as shows in Figure 1. Each element of the ADDIE model is a critical step, where the instructional designer makes crucial decisions to deliver effective instructions. Table 1 defines each of the five elements of ADDIE model [7]. This guideline is very flexible for building a road map for the entire training project. The ADDIE model is the most commonly used model and can be applied to almost every learning application [8] [9]. However, these elements of ADDIE model cannot be directly applied for human robot interaction system due to its architecture, autonomous nature and dynamically changing environment.

\subsection{ADDIE Model for HRI}

In this subsection, we modify the elements of ADDIE model so as to make them more applicable to human robot interaction in soccer robotics domain. We modified the elements thru independent review based on the humanoid soccer league objectives by a panel of four researchers working in interaction design and humanoid soccer robotics areas at our lab.

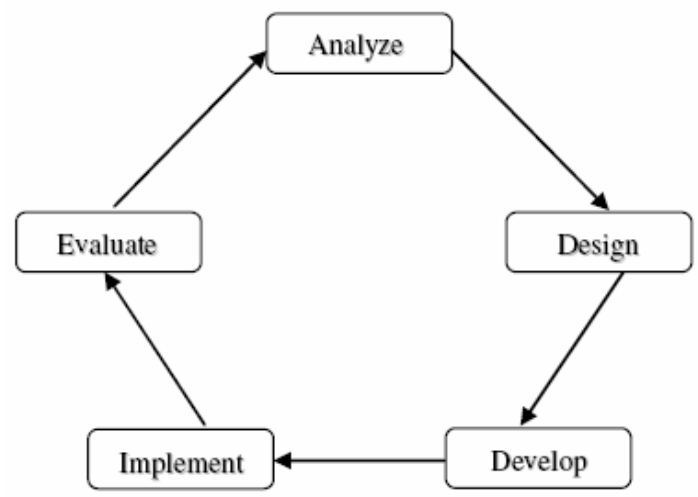

Fig. 1. ADDIE Instructional Design Model 
Table 1. Elements of ADDIE model

\begin{tabular}{|c|c|}
\hline No. & ADDIE Model \\
\hline 1. & $\begin{array}{l}\text { Analysis } \\
\text { In the analysis element, the instructional problem is validated, the instructional } \\
\text { goals and objectives are specified and the learning environment, learner's behav- } \\
\text { iour and skills are studied. The designer develops a perfect understanding of the } \\
\text { gaps between the desired outcomes and the present condition. Some common } \\
\text { questions analyzed in this element include, } \\
\text { 1. Who is the audience? } \\
\text { 2. What do they learn? } \\
\text { 3. What are the delivery options? } \\
\text { 4. What is the timeline for project completion? } \\
\text { 5. What constraints exist? }\end{array}$ \\
\hline 2. & $\begin{array}{l}\text { Design } \\
\text { In the design element, the designer selects and documents the specific learning } \\
\text { objectives, evaluation tools, lesson plan and delivery tools. The design process } \\
\text { must be both systematic and specific. Some common steps in design element } \\
\text { includes, } \\
\text { 11. Documentation of instructional design strategies } \\
\text { 12. Design user interface and experience } \\
\text { 13. Create prototype design materials }\end{array}$ \\
\hline 3. & $\begin{array}{l}\text { Develop } \\
\text { In the develop element, the designer completes the actual creation of the learning } \\
\text { materials designed to meet the learning objectives. A detailed action plan specify- } \\
\text { ing step by step procedure for implementation of the instructional strategy is pre- } \\
\text { pared. Designers work to develop technologies, perform debugging, review and } \\
\text { revise strategies. }\end{array}$ \\
\hline 4. & $\begin{array}{l}\text { Implement } \\
\text { In the implement element, the designer delivers the instructions to the target } \\
\text { group. In this element, training is provided to the facilitator on the course curricu- } \\
\text { lum, learning outcomes, method of delivery and evaluation procedures. The de- } \\
\text { livery environment should be prepared for implementation of the course. }\end{array}$ \\
\hline 5. & $\begin{array}{l}\text { Evaluate } \\
\text { In the evaluate element, the effectiveness of the instructional content and the } \\
\text { achievement of learning objectives are evaluated. Evaluation is administered in } \\
\text { two phases: formative and summative. Formative evaluation is performed within } \\
\text { each element of the ADDIE process whereas the summative evaluation is per- } \\
\text { formed after the implementation to determine its effectiveness in satisfying in- } \\
\text { structional objectives. }\end{array}$ \\
\hline
\end{tabular}

Table 2 defines the elements of modified ADDIE model for human robot interaction system. All the five elements of ADDIE remains with only modifications introduced to the definition of the elements. The modified ADDIE model was adopted for the development of human robot interaction for our soccer playing Robo-Erectus Junior humanoid robot. 
Table 2. Modified ADDIE Model

\begin{tabular}{|c|c|}
\hline No. & Modified ADDIE Model \\
\hline 1. & $\begin{array}{l}\text { Analysis } \\
\text { In the analysis element, the human robot interaction problem is validated, the human } \\
\text { robot interaction goals and objectives are specified, the application environment, } \\
\text { and robot operator's requirements are studied. The designer develops a perfect un- } \\
\text { derstanding of the gaps between the desired outcomes and the present condition. } \\
\text { Some common questions analyzed in this element include, } \\
\text { 1. What is the application domain? } \\
\text { 2. What are the requirements on human robot interaction? } \\
\text { 3. What are the control modalities (joystick/computer, etc)? } \\
\text { 4. What is the timeline for project completion? } \\
\text { 5. What constraints exist? }\end{array}$ \\
\hline 2. & $\begin{array}{l}\text { Design } \\
\text { In the design element, the designer selects and documents the specific human robot } \\
\text { interaction objectives, evaluation tools, and human robot interaction plan and con- } \\
\text { trol modalities. The design process must be both systematic and specific. Some } \\
\text { common steps in design element includes, } \\
\text { 11. Documentation of human robot interaction design strategies } \\
\text { 12. Design user interface and experience } \\
\text { 13. Create prototype human robot interaction designs }\end{array}$ \\
\hline 3. & $\begin{array}{l}\text { Develop } \\
\text { In the develop element, the designer completes the actual creation of the human } \\
\text { robot interaction to meet the interaction objectives. A detailed action plan specifying } \\
\text { step by step procedure for implementation of the human robot interaction strategy is } \\
\text { prepared. Designers work to develop technologies, perform debugging, review and } \\
\text { revise strategies. }\end{array}$ \\
\hline 4. & $\begin{array}{l}\text { Implement } \\
\text { In the implement element, the designer delivers the human robot interaction to the } \\
\text { robot operator. In this element, training is provided to the robot operator on the us- } \\
\text { age of the human robot interface, shortcuts, and help details. The robot operator } \\
\text { adopts the ADDIE modelled human robot interaction to operate the robot. }\end{array}$ \\
\hline 5. & $\begin{array}{l}\text { Evaluate } \\
\text { In the evaluate element, the effectiveness of the human robot interaction and the } \\
\text { achievement of human robot interaction objectives are evaluated. Evaluation is ad- } \\
\text { ministered in two phases: formative and summative. Formative evaluation is } \\
\text { performed within each element of the ADDIE process whereas the summative } \\
\text { evaluation is performed after the implementation to determine its effectiveness in } \\
\text { satisfying human robot interaction objectives. }\end{array}$ \\
\hline
\end{tabular}

\section{Robo-Erectus Junior - A Humanoid}

This section describes the Robo-Erectus Junior humanoid robot and its ADDIE modelled human robot interaction systems. The Robo-Erectus project is developed in the Advanced Robotics and Intelligent Control Centre of Singapore Polytechnic. RoboErectus Junior is one of the foremost leading soccer playing humanoid robots in the 


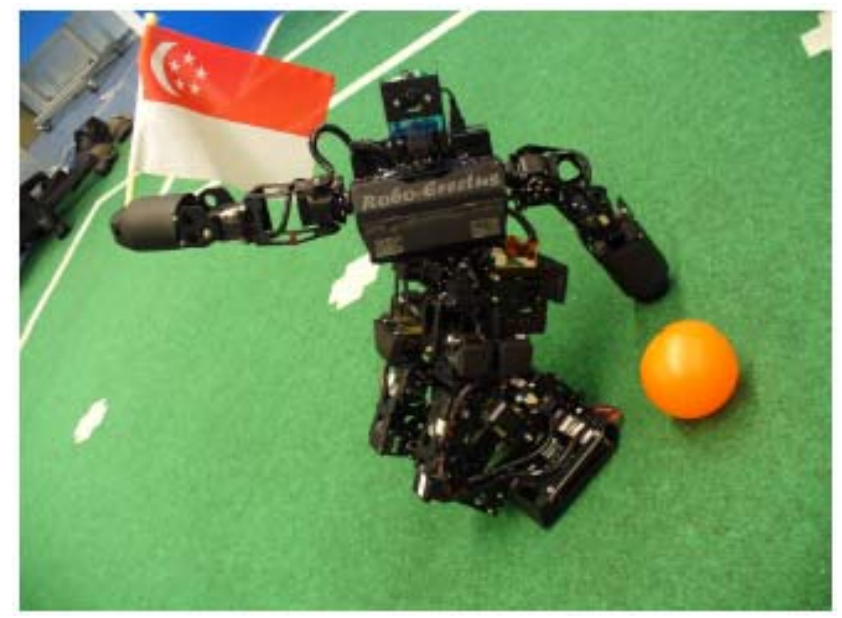

Fig. 2. Robo-Erectus Junior, the Latest Generation of the Family Robo-Erectus

RoboCup Humanoid League. Robo-Erectus Junior won the 2nd place in the Humanoid Walk competition at the RoboCup 2002 and got 1st place in the Humanoid Free Performance competition at the RoboCup 2003. In 2004, Robo-Erectus Junior won the 2nd place in Humanoid Walk, Penalty Kick, and Free Performance. The aim of the Robo-Erectus Junior development team is to develop a low-cost humanoid platform. The development of Robo-Erectus Junior has gone through many stages either in the design of its mechanical structure, electronic control system and gait movement control. Figure 2 shows the physical design of Robo-Erectus Junior. Robo-Erectus Junior has been designed to cope with the complexity of a 2 versus 2 soccer game. It has three processors each for vision, artificial intelligence and control. Table 3 shows the specification of the processors used in Robo-Erectus Junior. The platform is equipped with three sensors: an USB camera to capture images, a tilt sensor to detect a fall, and a compass to detect their direction [10]. To communicate with its teammates,

Table 3. Processor Specification of Robo-Erectus Junior

\begin{tabular}{|c|l|l|l|}
\hline Features & $\begin{array}{c}\text { Artificial } \\
\text { Intelligence } \\
\text { Processor }\end{array}$ & $\begin{array}{c}\text { Vision } \\
\text { Processor }\end{array}$ & \multicolumn{1}{c|}{$\begin{array}{c}\text { Control } \\
\text { Processor }\end{array}$} \\
\hline Processor & $\begin{array}{l}\text { Intel ARM } \\
\text { Xscale }\end{array}$ & $\begin{array}{l}\text { Intel ARM } \\
\text { Xscale }\end{array}$ & $\begin{array}{l}\text { ATMEL } \\
\text { ATmega-128 }\end{array}$ \\
\hline Speed & $400 \mathrm{Mhz}$ & $400 \mathrm{Mhz}$ & $16 \mathrm{Mhz}$ \\
\hline Memory & $16 \mathrm{MB}$ & $32 \mathrm{MB}$ & $4 \mathrm{~KB}$ \\
\hline Storage & $16 \mathrm{MB}$ & $16 \mathrm{MB}$ & $132 \mathrm{~KB}$ \\
\hline Interface & $\mathrm{RS} 232, \mathrm{WIFI}$ & RS232, USB & RS232, RS485 \\
\hline
\end{tabular}


Table 4. Physical Specification of Robo-Erectus Junior

\begin{tabular}{|c|c|c|c|c|}
\hline \multirow{2}{*}{ Weight } & \multicolumn{3}{|c|}{ Dimension } & Speed \\
\cline { 2 - 5 } & Height & Width & Depth & Walking \\
\hline $3.2 \mathrm{Kg}$ & $480 \mathrm{~mm}$ & $270 \mathrm{~mm}$ & $150 \mathrm{~mm}$ & $2 \mathrm{~m} / \mathrm{min}$ \\
\hline
\end{tabular}

Robo-Erectus Junior uses a wireless network connected to the artificial intelligence processor. The vision processor performs recognition and tracking of objects of interest including ball, goal, field lines, goal post teammate and the opponents based on a blob finder based algorithm. The further processing of detected blobs, wireless communications and decision making are performed by the artificial intelligence processor which selects and implements the soccer skills (like walk to the ball, pass ball, kick ball, dive....) the robot is to perform. Finally, the control processor handles the low level control of motor based on the soccer skill selected by the artificial intelligence processor. Robo-Erectus Junior was fabricated to participate in RoboCup 2007 in the KidSize category. Table 4 shows the physical specifications of Robo- Erectus Junior. It is powered by two high-current Lithiumpolymer rechargeable batteries, which are located in each foot. Each battery cell has a weight of only $110 \mathrm{~g}$ providing $12 \mathrm{v}$ which means about 15 minutes of operation [11].

In the RoboCup 2007 competitions, Robo-Erectus Junior participated in the 2 versus 2 Soccer Games and the Technical Challenges.

\subsection{Human Robot Interaction System}

Figure 3 shows the human robot interaction system of Robo-Erectus Junior, containing wealth of information. A separate window shows the video streaming from the robot. On the lower right of the interface is the score board showing the current status of the soccer match to the left of which is the simulated actual match with the field localized Robo-Erectus Junior and its team mates. On the top of the interface is the control buttons for team configuration and game status signals.

The human robot interaction design was developed to be homogeneous so as to adopt the same interaction design for multiple robot platforms. The user can view and operate both the team configuration controls and game status signal controls at the same time. The team configuration controls include options for assigning robot identification numbers to identify each and individual robots in own team as well as the opponent team, goal colours to identify own and opponent goals, and individual roles of robots like striker, goalie or hybrid. In striker mode, the robot aims to score goals by kicking the ball to the opponent goal. In goalie mode, the robot defends its goal area by stopping the ball from the opponent. In hybrid mode, the robot plays the role of striker as well as goalie depending on the circumstance. The team configuration controls also include options for viewing the sensor and actuator status which include display of sensor data and actuator position data.

The game status signal controls provide robot external information on the status of the match including kick off, time out, time off, resume, ball out, indirect kick and catch. Kick-off is a way of starting or restarting play at the start of the match, after a goal has been scored, at the start of the second half of the match, or at the start of each 


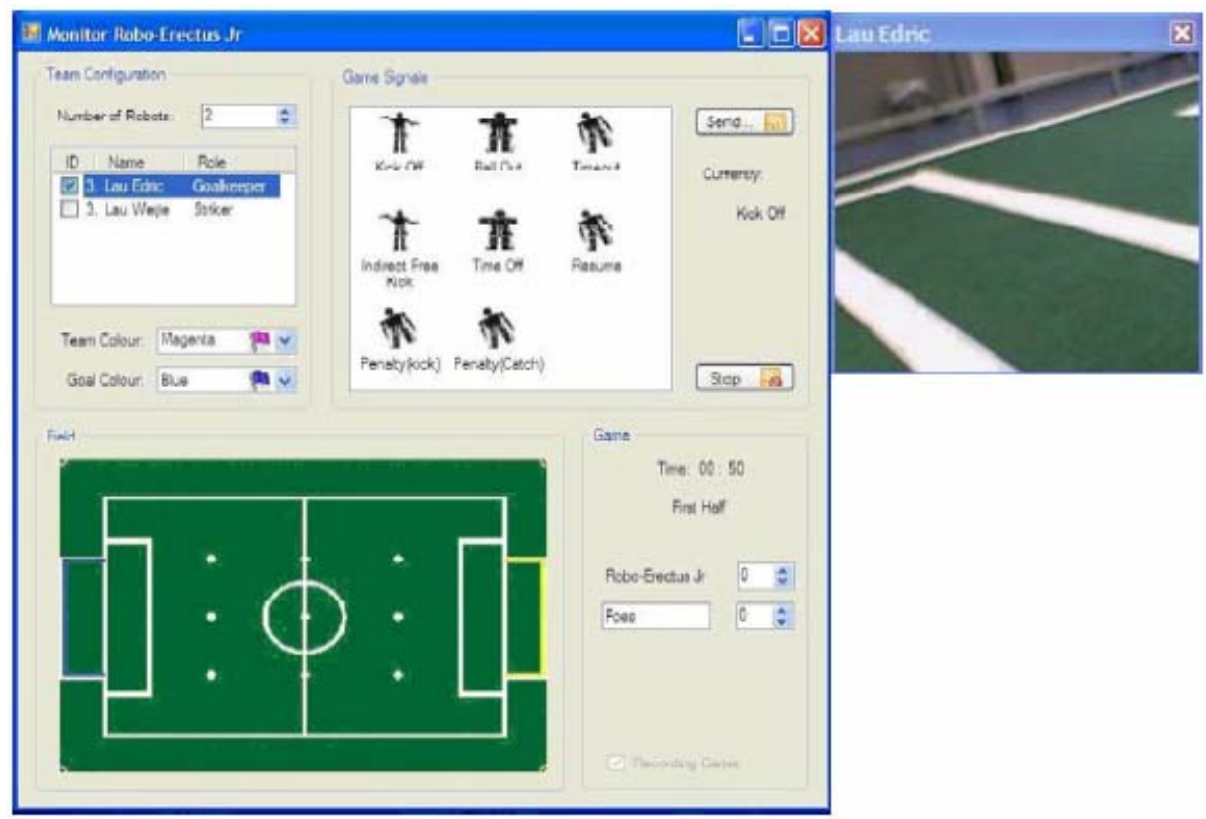

Fig. 3. Human Robot Interaction System of Robo-Erectus Junior Humanoid Robot

period of extra time. Depressing the kick off icon would start the robot in the autonomous mode for a soccer game. A team may request for timeout period of 120 s to service their robots for software/hardware faults during a soccer match causing a stoppage of the game. Each team may take atmost one timeout per half period of the match. Depressing the timeout icon would perform the test routine for the checking software faults with the artificial intelligence algorithms of the humanoid robot.

Time off indicates the half time period break in the middle of the soccer game. Depressing the timeoff icon pause the robot's status and depressing the resume icon would start the robot from pause state into autonomous soccer playing mode. An indirect free kick is awarded to the opposing team if a player commits any offenses in a manner considered by the referee to be careless, reckless or using excessive force. Depressing the indirect kick icon would enable the robot to start in autonomous soccer playing mode with an indirect free kick. Depressing the catch icon would perform a routine check to test the goalie robot's ability to dive for catching an incoming ball. The controls under the game control signal can also be used to test the functionality of the robot hardware (sensors, onboard controllers, joints and actuators) and software (implemented algorithms).

\section{Experimental Results}

The modified ADDIE model was adopted for the development of human robot interaction for our soccer playing Robo-Erectus Junior humanoid robot. In the analysis stage, the human robot interaction goal was determined for 2 versus 2 humanoid 
Table 5. Human Robot Interaction Requirements of Robot Operators

\begin{tabular}{|c|l|}
\hline No. & Human Robot Interaction Requirements of Robot Operators \\
\hline 1 & Simple to use \\
\hline 2 & Visibility of system status \\
\hline 3 & Match between system and the real world \\
\hline 4 & Prioritized placement of controls \\
\hline 5 & Help operators to recognize, diagnose, and recover from errors. \\
\hline
\end{tabular}

Table 6. Objectives for Human Robot Interaction

\begin{tabular}{|c|l|}
\hline No. & Objectives for Human Robot Interaction \\
\hline 1 & Detect software fault \\
\hline 2 & Detect locomotion fault \\
\hline 3 & Detect sensor \& actuator fault \\
\hline
\end{tabular}

soccer leagues by expert researchers as, Human robot interaction design must be able to detect the system fault in the robot within 60s of the timeout period. A survey was conducted with the robot operators on their requirements for the human robot interaction. Table 5 shows the key requirements specified by the robot operators. The human robot interaction objectives were derived from the interaction goal as shown in Table 6 . In the design stage, a blueprint detailing interaction objectives, evaluation tools, and human robot interaction plan and control modalities was created. Interaction strategy was then developed for localization of video displays, sensor displays, and control buttons. Computer keyboard control was chosen as the only control modality. One to one and survey based formative evaluations were conducted at the end of each of the five stage of ADDIE model. RoboCup 2 versus 2 humanoid soccer league mimicked soccer matches were selected for summative evaluation of the human robot interaction design. In the develop stage, the actual human robot interaction for soccer playing humanoid robot was created. Three experienced research staffs from our lab selected the appropriate interaction components and developed the human robot interaction design for our soccer playing humanoid robot, Robo-Erectus Junior. In the implementation stage, the human robot interaction was passed to the robot operator. The robot operator was also trained on the effective use of the developed human robot interaction.

For summative evaluations, we conducted 20 RoboCup 2 versus 2 humanoid soccer league mimicked soccer matches with other teams in our lab. Each match had two equal periods of 10 minutes. Players were entitled to an interval at half-time. Results of the experiment showed that 41 faults occurred during 20 soccer matches. There were 7 faults due to artificial intelligence algorithm and 34 faults due to locomotion related issues. In all the 41 cases, the fault was detected without having to make a replacement of the robot within 60 s of the timeout period. Figure 4 shows the 41 faults and their corresponding fault detection time period with the ADDIE modelled human robot interaction. 


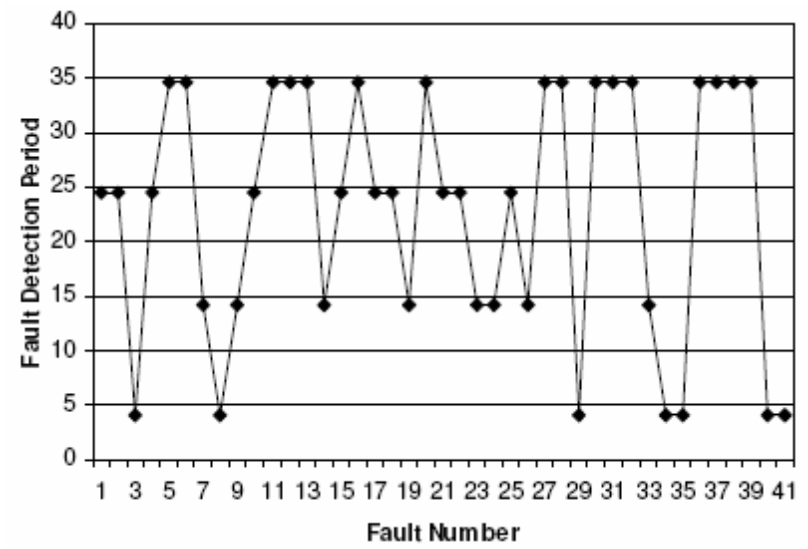

Fig. 4. Fault Detection Period Versus Fault Number

Table 7. Software and Hardware Faults \& Their Average Fault Detection Time Periods

\begin{tabular}{|c|c|c|c|}
\hline $\begin{array}{c}\text { Type of } \\
\text { Fault }\end{array}$ & $\begin{array}{c}\text { Total Number } \\
\text { of Soccer } \\
\text { Matches }\end{array}$ & $\begin{array}{c}\text { Number } \\
\text { of faults }\end{array}$ & $\begin{array}{c}\text { Average Fault } \\
\text { Detection Periods (s) }\end{array}$ \\
\hline Software & 20 & 13 & $8.78 \mathrm{~s}$ \\
\hline Hardware & 20 & 28 & $29.48 \mathrm{~s}$ \\
\hline
\end{tabular}

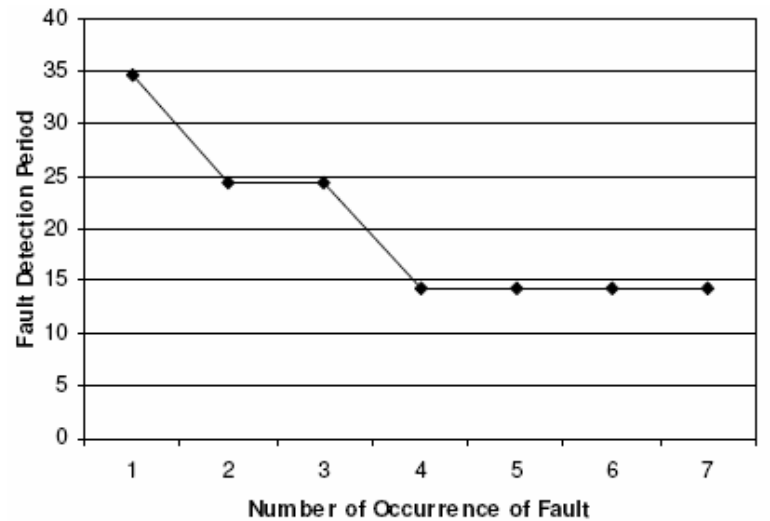

Fig. 5. Fault Detection Period Versus Number of Occurrence of the Knee Joint Fault

The average fault detection time was found to be $22.91 \mathrm{~s}$. Table 7 shows the number of software and hardware faults and their average fault detection time periods.

Experiments with the unmodelled human robot interaction design showed that the average fault detection time was found to be 57.47s. Therefore, the ADDIE model helped in improving the fault detection performance of human robot interaction in 
soccer domain by $250.85 \%$. It was also found from the experiments that time period for detecting repeating faults tend to decrease with number of occurrence of that particular fault. For example, the fault due to heated foot actuator occurred 6 times during the soccer matches. Figure 5 shows the reduction in the time period for detection of fault due to heated foot actuator as the number of times of occurrence of that fault increases. The ADDIE modelled human-humanoid robot interaction of Robo-Erectus Junior was adopted for RoboCup 2007 humanoid soccer league at Atlanta, USA.

\section{Conclusion}

We have presented the application of ADDIE model for development of human robot interaction in soccer domain. ADDIE modelled human robot interaction was able to detect software/hardware faults in $100 \%$ of the cases within 60 s of the timeout period during the soccer matches. The average fault detection time period for the ADDIE modelled interaction design was found to be $22.91 \mathrm{~s}$ as compared to $57.47 \mathrm{~s}$ with the unmodelled interaction design improving the performance. ADDIE model provides a systematic approach for human robot interaction development that can be readily adopted by soccer robotics community with ease.

\section{References}

1. Metev, S.M., Veiko, V.P.: Task Structure and User Attributes as Elements of HumanRobot Interaction Design. In: Proc. IEEE RO-MAN 2006 Conference, Hatfield, United Kingdom (2006)

2. Weiss, N., Hildebrand, L.: An exemplary robot soccer vision. In: CLAWAR/EURON Workshop on Robots in Entertainment, Leisure and Hobby, Vienna, Austria (December 2004)

3. Calderon, C.A., Zhou, C., Yue, P.K., Wong, M., Elara, M.R.: A distributed embedded control architecture for humanoid soccer robots. In: Proceeding of CLAWAR Conference 2007, Singapore (2007)

4. Hu, L., Zhou, C., Sun, Z.: Estimating probability distribution with Q-learning for biped gait generation and optimization. In: Proc. Of IEEE Int. Conf. on Intelligent Robots and Systems (2006)

5. [Online] http://itsinfo.tamu.edu/workshops/handouts/pdf_handouts/ addie

6. RoboCup, Compiled by Emanuele Menegatti: RoboCup Soccer Humanoid League Rules and Setup for the 2007 competition in Atlanta, USA (2007),

http: / / www. robocup.org

7. [Online] http://en.wikipedia.org/wiki/ADDIE_Model

8. [Online]

http: / / www.syl.com/hb/

instructionaldesignersmodelstheaddiemodel.html

9. [Online] http://www.e-learningguru.com/articles/art2_1.html

10. Zhou, C., Yue, P.K.: Robo-Erectus: A low cost autonomous humanoid soccer robot. Advanced Robotics 18(7), 717-720 (2004)

11. Team Robo-Erectus website (2007), http: / /www . robo-erectus . org 